Kumberger, O., Riede, J.,

Schmidbaur, $\mathrm{H}$.

Orotat-Komplexe, II. - Herstellung und Kristallstrukturen von Calcium- und Zink-orotat(2-)-hydraten

2743

Mikulcik, P., Riede, J., Schmidbaur, $\mathrm{H}$.

Kreißl, F. R., Stegmair, C. M.
Metall-Ionen-Fixierung durch Dipeptide: Struktur des durch „Aspartam“-Hydrolyse gebildeten Produkts, Natrium-cyclo(L- $\alpha$-aspartyl-L-phenylalanin)-tetrahydrats, $\mathrm{Na}[c$ (L-Asp-L-Phe) $] \cdot 4 \mathrm{H}_{2} \mathrm{O}$

2747 Carbonyl $\left(\eta^{5}\right.$-cyclopentadienyl)(trimethylphosphan) $\left[\eta^{2}\right.$-(methylthio)phenylcarben $]-$ molybdän-tetrafluoroborat - ein amphiphiler Carbenkomplex
Orotate Complexes, II. - Preparation and Crystal Structures of Calcium and Zinc Orotate $(2-)$ Hydrates

\section{Querverweise / Cross References}

Regitz, M. et al.

Grevels, F.-W., Özkar, S. et al.
2857
Organophosphorverbindungen, 51

Photoinduzierte Reaktionen von $\mathrm{Cr}(\mathrm{CO})_{3}$ koordiniertem 1,3,5-Cycloheptatrien
Metal Ion Binding by Dipeptides: Structure of the Product Generated in "Aspartame" Hydrolysis, Sodium cyclo(L- $\alpha$-Aspartyl-L-phenylalanine) Tetrahydrate, $\mathrm{Na}$ [c(L-Asp-L-Phe) $] \cdot 4 \mathrm{H}_{2} \mathrm{O}$

Carbonyl $\left(\eta^{5}\right.$-cyclopentadienyl)(trimethylphosphane) $\left[\eta^{2}\right.$-(methylthio)phenylcarbene]molybdenum Tetrafluoroborate - an Amphiphilic Carbene Complex

Organophosphorus Compounds, 51

- Photoinduced Reactions of $\mathrm{Cr}(\mathrm{CO})_{3}-\mathrm{Co}-$ ordinated 1,3,5-Cycloheptatriene
Roth, W. R., Langer, R.,

Ebbrecht, T., Beitat, A.,

Lennartz, H.-W.

Bestmann, H. J., Schaper, W., Ruppert, D., Zimmermann, R., Burzlaff, $\mathrm{H}$.

Bestmann, H. J., Schaper, W., Holzmann, H. G., Zimmermann, R.

Liebscher, J., Riemer, B.,

Hassoun, A., Stößer, R.,

Grubert, L.

Ohm, S., Bäuml, E.,

Mayr, $\mathrm{H}$.

Reichardt, C., Schäfer, G., Allmann, R., Krestel, M.,

Knecht, J., Gärtner, R.

Schulz, J., Nieger, M., Vögtle, F.

Sustmann, R., Korth, H.-G., Nüchter, U., Siangouri-Feulner, I., Sicking, W.

Auchter-Krummel, P.,

Krummel, G.,

Lex, J., Müllen, K.

Schade, P., Schäfer, T., Müllen, K., Bender, D.,

Knoll, K., Bronstert, K.

Fuchs, E., Breit, B., Heydt, H., Schoeller, W., Busch, T., Krüger, C., Betz, P., Regitz, M.
2751 Zur Energie-Delle von Diradikalen, III. 2,3,5,6-Tetramethylen-1,4-cyclohexadiyl

2761 Phosphinalkylene, 49. - Chemie und Stereochemie des 9,10,19,20-Tetrahydrotetrabenzo $[a, c, g, i]$ cyclododecens und einiger seiner Derivate

2773 Synthese und Reaktionen der Stereoisomeren von Tetrabenzo- und Tetranaphtho[ $[a, c, g, i]$ cyclododecen

2781 Synthese neuartiger Bispyrimido[1,2-b: $\left.1^{\prime}, 2^{\prime}-e\right][1,2,4,5]$ tetrazine

2785 Eine vielseitige Synthese von 1,4-Dienen: Verwendung von Vinylethern als VinylKation-Äquivalente

2791 Über die Konformation eines trinuclearen [1.1.1]Tetramethinium-Cyaninfarbstoffs mit Indolin-Endgruppen

2797 Regioselektive Komplexierung von [2.2]Metacyclophanen mit Triammintricarbonylchrom

2811 Sind Charge-Transfer-Komplexe Zwischenstufen bei Diels-Alder-Reaktionen? Eine Fallstudie über die Reaktion von 1,2Dimethylencyclopentan mit Tetracyanethylen

2819 Reduktive Umwandlungen, 18. - Cycloannellierung und Überbrückung von Cyclooctatetraen

2833 Reduktive Umwandlungen, 19. - Elektron-Transfer-induzierte Dimerisierung von $\beta$-Alkylstyrolen und die Strukturen der resultierenden 1,4-Dilithiobutane

- Organophosphorverbindungen, 51. Phosphatriafulvene - Phosphaalkene mit inverser Elektronendichte
Energy Well of Diradicals, III. - 2,3,5,6Tetramethylene-1,4-cyclohexadiyl

Phosphine Alkylidenes, 49. - Chemistry and Stereochemistry of $9,10,19,20$-Tetrahydrotetrabenzo[a,c,g,i]cyclododecene and of Some Derivatives

Synthesis and Reactions of the Stereoisomers of Tetrabenzo- and Tetranaphtho $[a, c, g, i]$ cyclododecene

- Synthesis of Novel Bispyrimido[1,2-b: $\left.1^{\prime}, 2^{\prime}-e\right][1,2,4,5]$ tetrazines

A Versatile Synthesis of 1,4-Dienes: Use of Vinyl Ethers as Vinyl Cation Equivalents

Conformation of a Trinuclear [1.1.1]Tetramethinium Cyanine Dye with Indoline End Groups

Regioselective Complexation of [2.2]Metacyclophanes with Triamminetricarbonylchromium

Are Charge-Transfer Complexes Intermediates in Diels-Alder Reactions? A Case Study of the Reaction of 1,2-Dimethylenecyclopentane with Tetracyanoethylene

Reductive Transformations, 18. - Cycloannulation and Bridging of Cyclooctatetraene

Reductive Transformations, 19. - Electron-Transfer-Induced Dimerization of $\beta$ Alkylstyrenes and the Structures of the Resulting 1,4-Dilithiobutanes

Organophosphorus Compounds, 51. Phosphatriafulvenes - Phosphaalkenes with Inverse Electron Density 
Inhalt (Fortsetzung)

Fischler, I., Grevels, F.-W., Leitich, J., Özkar, S.

2857

Photoinduzierte Reaktionen von $\mathrm{Cr}(\mathrm{CO})_{3}$ koordiniertem 1,3,5-Cycloheptatrien: $[6+2]-C y c l o a d d i t i o n$ mit einem Alkin und katalytische 1,6-Hydrierung

Elsässer, D., Hassenrück, K.,

Martin, H.-D., Mayer, B., Lutz, G., Prinzbach, H.

Albert, B., Elsässer, D., Martin, H.-D., Mayer, B., Chow, T. J., Marchand, A. P., Ren, C.-T., Paddon-Row, M. N.

Huda, E., Martin, H.-D., Mayer, B., Somnitz, K.-H. Steigel, A., Haddad, H., Distefano, G., Modelli, G.

Beck, A. K., Seebach, D.
Kleine und mittlere Ringe, 77. - Sich der klassischen $\pi, \pi$-Konjugation nähernde nichtkonjugierte $\pi, \pi$-Wechselwirkungen - PE-spektroskopische Untersuchungen des 7,8;12,19-Diseco-1,16-dodecahedradien-System

Kleine und mittlere Ringe, 78. - KäfigDimere von Norbornadien mit rechtwinkliger Anordnung von Subchromophoren: Orbital-Wechselwirkung im Heptacyclo$\left[6.6 .0 \cdot 0^{2,6} \cdot 0^{3,13} \cdot 0^{4,11} \cdot 0^{5,9} \cdot 0^{10,14}\right]$ tetradecan-System

Kleine und mittlere Ringe, 79. - 4-Azatetracyclo[3.3.0.0 $\left.0^{2,8} \cdot 0^{3,6}\right]$ octane: Neuer Heterocyclen durch Addition von Sulfonylaziden an 7-substituierte Norbornadiene - Experimentelle Bestätigung von $\sigma^{*}$-Wechselwirkungen im Norbornan-Gerüst

2897 Aldol- und Michael-Additionen fluorierter Nitroalkane an Aldehyde, Ketone und $\alpha, \beta$ ungesättigte Carbonylverbindungen

\section{Contents (Continued)}

Photoinduced Reactions of $\mathrm{Cr}(\mathrm{CO})_{3}-\mathrm{Co}-$ ordinated 1,3,5-Cycloheptatriene: [6+2] Cycloaddition with an Alkyne and Catalytic 1,6-Hydrogenation

Small and Medium Rings, 77. - Nonconjugated $\pi, \pi$ Interactions Approaching Classical $\pi, \pi$ Conjugation - PE-Spectroscopical Investigation of the 7,8;12,19-Diseco-1,16-dodecahedradiene System

Small and Medium Rings, 78. - Cage Dimers of Norbornadiene with Perpendicular Arrangement of Subchromophores: Orbital Interaction in the Heptacyclo$\left[6 \cdot 6 \cdot 0 \cdot 0^{2,6} \cdot 0^{3,13} \cdot 0^{4,11} \cdot 0^{5,9} \cdot 0^{10,14}\right]$ tetradecane System

Small and Medium Rings, 79. - 4-Azatetracyclo[3.3.0.0 $0^{2,8} \cdot 0^{3,6}$ ]octanes: New Heterocycles by Addition of Sulfonyl Azides to 7-Substituted Norbornadienes - Experimental Verification of $\sigma^{*}$ Interactions in the Norbornane Skeleton

Aldol and Michael Additions of Fluorinated Nitroalkanes to Aldehydes, $\mathrm{Ke}$ tones, and $\alpha, \beta$-Unsaturated Carbonyl Compounds

\section{Notiz / Note}

Drewes, S. E., Malissar, D. G. S., Roos, G. H. P.

\section{Querverweis / Cross Reference}

Schmidbaur, H. et al.

2743
Kristalline, enantiomerenreine Aldole aus einem von (-)-Ephedrin abgeleiteten $N$ Acylimidazolidin-2-on

Metall-Ionen-Fixierung durch Dipeptide

Publikationssprache
Crystalline, Enantiomerically Pure Aldols from a (-)-Ephedrine-Derived $N$-Acylimidazolidin-2-one

Metal Ion Binding by Dipeptides

Language of Publication 


\title{
A Versatile Synthesis of 1,4-Dienes: Use of Vinyl Ethers as Vinyl Cation Equivalents $^{\not 3}$
}

\author{
Stefan Ohm, Englbert Bäuml, and Herbert Mayr* \\ Institut für Chemie, Medizinische Universität zu Lübeck, \\ Ratzeburger Allee 160, W-2400 Lübeck 1, F.R.G.
}

Received June 3, 1991

Key Words: Enol ether / Allylsilane / Bromo ether / 1,4-Diene / Alkoxyalkylation

The $\alpha, \beta$-dibromo ethers 2 , in situ generated from the vinyl ethers 1 and bromine, react with the allylsilanes 3 in the presence of $\mathrm{ZnCl}_{2}$ to give the $\beta$-bromo ethers 4. Treatment of 4 with $\mathrm{Na}$ in diethyl ether yields 1,4-dienes 5 in $62-79 \%$ overall yield.
Most syntheses of 1,4-dienes proceed by combination of vinylic nucleophiles with allylic electrophiles ${ }^{1}$, and examples for the coupling of a vinylic electrophile with an allylic nucleophile are rare ${ }^{1)}$. A general method for the synthesis of 1,4-dienes using the latter approach has recently been described by Lipshutz (Scheme 1) ${ }^{2}$.

Scheme 1

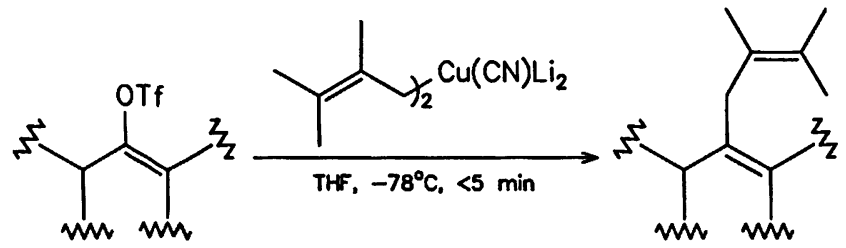

We now describe another example for constructing 1,4dienes from vinyl cation and allyl anion equivalents, where the readily available vinyl ethers are used as vinyl cation equivalents (Scheme 2).

Scheme 2<smiles></smiles>

For this purpose, the reaction sequence depicted in Scheme 3 has been elaborated. A comparison of structures $\mathbf{1}$ and $\mathbf{5}$ shows that, overall, the alkoxy group of vinyl ethers is replaced by an allylic group.

\section{Formation of the $\beta$-Bromo Ethers 4}

In view of the high tendency of vinyl ethers to undergo cationic polymerization, the 1,2-dibromo ethers 2 have been prepared by dropwise addition of the vinyl ethers 1 to so-

Scheme 3

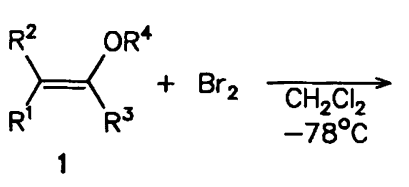<smiles>[R]C([R])(Br)C([R])(Br)Br</smiles>
2<smiles>[R]C([R])=C([R3])C([R])([R])[Si]C</smiles><smiles>[R]C([R])=C([R20])C([R])([R9])C([R])([R])C([R])([R])Br</smiles>

4

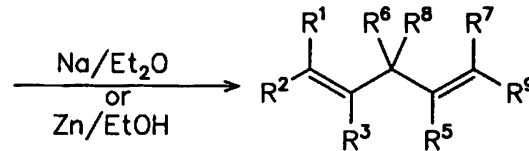

5 lutions of bromine in $\mathrm{CH}_{2} \mathrm{Cl}_{2}$ at $-78^{\circ} \mathrm{C}$. Even under these conditions a small excess of 1 over bromine is usually needed to achieve complete decolorization of the bromine solution (see Experimental). The highly reactive and presumably toxic dibromo ethers 2 have not been isolated, and the $\beta$-bromo ethers 4 have been produced in a one-pot reaction by adding $\mathrm{ZnCl}_{2}-\mathrm{Et}_{2} \mathrm{O}^{3)}$ and the allylsilanes 3 to the dichloromethane solutions obtained by bromination of the vinyl ethers ${ }^{4)}$. Compounds 4 have either been purified by distillation or immediately converted into the 1,4-dienes 5 .

All combinations of enol ethers and allylsilanes examined in this work gave the $\beta$-bromo ethers 4 in good yield. Contaminations observed in some runs were due to the use of moist bromine, leading to $\mathrm{HBr}$ adducts of the vinyl ethers, which then reacted with the allylsilanes 3 to give debrominated analogs of 4 (e.g. 7). As the stereochemistry of the intermediate bromo ethers 4 is usually not important for the structure of the elimination products 5 , the diastereoselectivities of the $\mathrm{C}-\mathrm{C}$ bond-forming reactions have generally not been investigated. Only in the case of $4 \mathrm{c}, \mathrm{a}^{13} \mathrm{C}$ NMR spectrum has been taken, which showed the highly selective formation of one diastereomer $(\approx 95: 5)$ from a $2: 1$ mixture of diastereomeric dibromo ethers $\mathbf{2}$. 


\section{Elimination Reactions}

The production of alkenes by $\mathrm{Zn}$-promoted elimination of halogen and alkoxyl from $\beta$-halogeno ethers has long been known ${ }^{5}$. The reaction conditions were optimized for the conversion $\mathbf{4 d} \rightarrow \mathbf{5 d}$, and it was found that a mixture of $4 \mathrm{~d}$ and 5 equivalents of zinc powder in ethanol or methanol had to be refluxed for approximately 20 hours to achieve complete consumption of the bromo ether. A slight retardation of the elimination was observed, when 4 mol-\% of $\mathrm{ZnCl}_{2}$ was added to the reaction mixture.

Table 1. 1,4-Dienes 5 from vinyl ethers 1 and allylsilanes 3

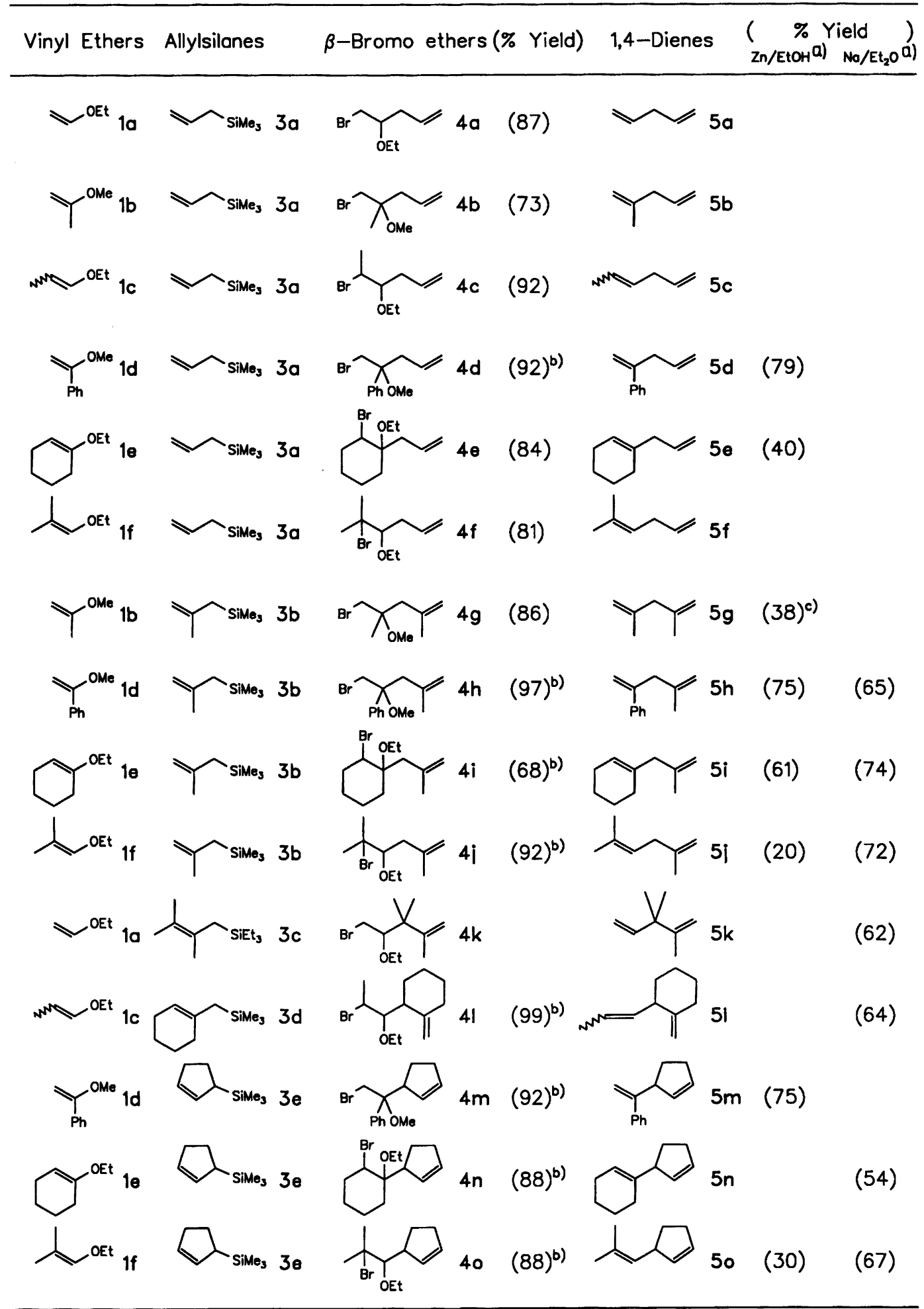

a) Yield with respect to vinyl ethers 1 and allylsilanes 3. - b) Yield of crude material. - ${ }^{\text {c) }}$ Ref. ${ }^{10)}$. 
W hile the elimination with zinc in alcohols gave satisfactory results in several cases (Table 1), there were systems, where generally high amounts of side products were observe $d$ under these conditions. Treatment of 40 with zinc dust (Riedel-de Haën) in ethanol, for example, gave 5o, 6, and 7 (Scheme 4) in a 58:33:9 ratio (GC), and zinc powder (Mer ck) in ethanol yielded these compounds in a 72:22:6 ratio. Heating of 40 with a $\mathrm{Zn} / \mathrm{Cu}$ couple (Merck) even led to the predominant formation of $6(50: 6: 7=38: 46: 16)$. While no reaction was observed when 40 was treated with magn esium turnings in diethyl ether, the analogous treatment with sodium wire led to the selective formation of comp ound $50^{6}$.

Schem e 4

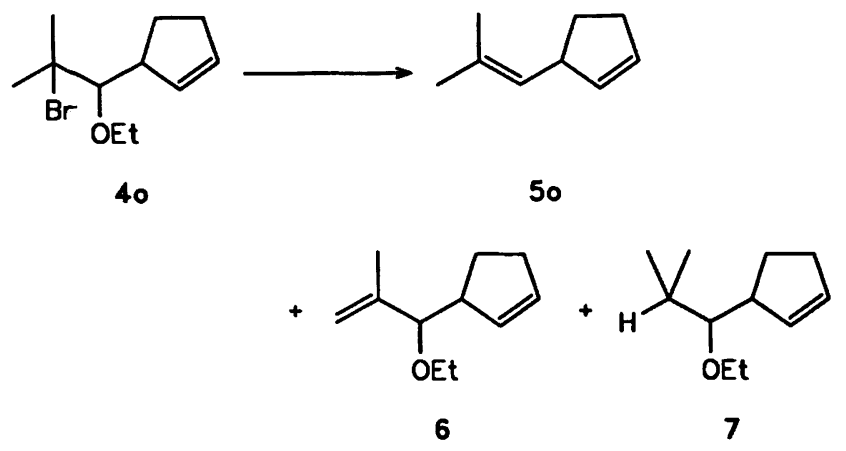

As this method gave good yields of 1,4-dienes in all cases examined, the elimination with $\mathrm{Na}$ in $\mathrm{Et}_{2} \mathrm{O}$ is the method of choice for further applications.

\section{Conclusion}

Scheme 3 provides a straightforward synthesis for 1,4dienes with variable substitution pattern. This method may be considered as a variant of the Boord olefin synthesis (Scheme 5),7), which has also been employed for the synthesis of 1,4-dienes by using allylic Grignard reagents ${ }^{8}$. As unsymmetrical allylmagnesium halides often do not react regioselectively, the advantage of the method presented in this article is not only the higher overall yield but also the greater regioselectivity of the $\mathrm{C}-\mathrm{C}$ bond-forming reaction.

Scheme 5

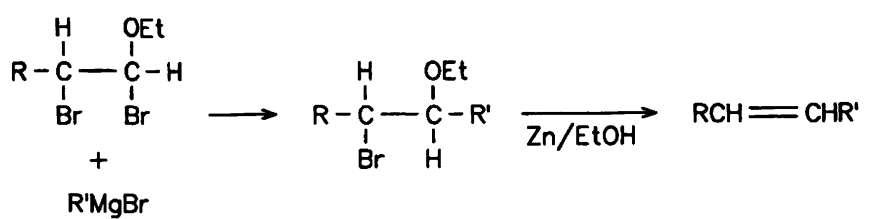

We thank $R$. Wendt for orientating experiments, the Deutsche Forschungsgemeinschaft and the Fonds der Chemischen Industrie for financial support. We also would like to express our sincere thanks to Profs. Tochtermann and Hoppe for accepting this work as a part of a Diplomarbeit at the Universität Kiel.

\section{Experimental}

'H NMR: EM 390 (Varian). - ${ }^{13} \mathrm{C}$ NMR: XL 200 (Varian). MS: $70-250 \mathrm{E}$ (VG-Instruments).
The enol ethers were commercially available $(1 \mathbf{a}-\mathbf{c})$, or were synthesized from the corresponding acetals by $\mathrm{H}_{3} \mathrm{PO}_{4}$-catalyzed alcohol elimination using literature procedures $(\mathbf{1 d}-\mathbf{f})^{9)}$. The allylsilanes $\mathbf{3 b}$ and $3 \mathbf{e}$ were obtained by magnesium-promoted coupling of the corresponding allyl chlorides with chlorotrimethylsilane ${ }^{10,11)}$, 3c was prepared by photochemically induced hydrosilation of 2,3dimethyl-1,3-butadiene in the presence of $\mathrm{Cr}(\mathrm{CO})_{6}{ }^{12)}$ and $3 \mathrm{~d}$ from (trimethylsilyl)methylmagnesium chloride and 1-(trimethylsiloxy)1-cyclohexene in the presence of catalytic amounts of nickel acetylacetonate $^{13)}$.

Typical Procedure for the Synthesis of the B-Bromo Ethers 4: Bromine $(1.60 \mathrm{~g}, 10.0 \mathrm{mmol})$ was dissolved in $\mathrm{CH}_{2} \mathrm{Cl}_{2}(20 \mathrm{ml})$ in a 100 $\mathrm{ml}$ flask and the solution cooled at $-78^{\circ} \mathrm{C}$. A solution of the enol ether $1 \mathrm{e}(1.39 \mathrm{~g}, 11.0 \mathrm{mmol})$ in $\mathrm{CH}_{2} \mathrm{Cl}_{2}(20 \mathrm{ml})$ was added dropwise with stirring, leading to complete decolorization of the solution. Allylsilane $3 \mathrm{a}(1.26 \mathrm{~g}, 11.0 \mathrm{mmol})$ and $\mathrm{ZnCl}_{2}-\mathrm{Et}_{2} \mathrm{O}$ [4.8 mmol; $2.5 \mathrm{ml}$ of a solution obtained by dissolving $\mathrm{ZnCl}_{2}(50 \mathrm{~g})$ in diethyl ether $(60 \mathrm{ml})$ and dichloromethane $(120 \mathrm{ml})]^{3)}$ were added, and the mixture was kept at $-78^{\circ} \mathrm{C}$ for $2 \mathrm{~h}$. The solution was washed with two 35-ml portions of $25 \%$ aqueous $\mathrm{NH}_{4} \mathrm{Cl}$ and dried with $\mathrm{MgSO}_{4}$. After evaporation of the solvent, spectroscopically pure 1-allyl-2bromo-1-ethoxycyclohexane (4e) was obtained in $84 \%$ yield $(2.29 \mathrm{~g})$. - ${ }^{1} \mathrm{H}$ NMR (90 MHz, CDCl $\left.)_{3}\right): \delta=1.05-2.78(\mathrm{~m}, 13 \mathrm{H}), 3.20-3.70$ $\left(\mathrm{m}, 2 \mathrm{H}, \mathrm{OCH}_{2} \mathrm{CH}_{3}\right), 4.10\left(\mathrm{~m}_{\mathrm{c}}, 1 \mathrm{H}, \mathrm{CHBr}\right), 5.0-5.4\left(\mathrm{~m}, 2 \mathrm{H},=\mathrm{CH}_{2}\right)$, $5.73\left(\mathrm{~m}_{\mathrm{c}}, 1 \mathrm{H}, \mathrm{CH}=\right)$.

Note: This procedure is recommended for all compounds 4 , though in the early phase of this project different amounts of the reactants have been used (see below).

5-Bromo-4-ethoxy-1-pentene (4a) was obtained in $87 \%$ yield $(1.17 \mathrm{~g})$ from $\mathrm{Br}_{2}(1.12 \mathrm{~g}, 7.01 \mathrm{mmol}), 1 \mathrm{a}(500 \mathrm{mg}, 6.93 \mathrm{mmol})$, $\mathrm{ZnCl}_{2}-\mathrm{Et}_{2} \mathrm{O}(1.75 \mathrm{ml}, 3.36 \mathrm{mmol})$, and $3 \mathrm{a}(1.20 \mathrm{~g}, 10.5 \mathrm{mmol})$ after a reaction time of $15 \mathrm{~h}$; b.p. $40-45^{\circ} \mathrm{C}$ (bath) $/ 2-5$ mbar [ref. ${ }^{14}$ $\left.81.8-84.0^{\circ} \mathrm{C}(33 \mathrm{mbar})\right]$. $-{ }^{1} \mathrm{H}$ NMR $\left(\mathrm{CCl}_{4}\right): \delta=1.20(\mathrm{t}, J=$ $\left.6.0 \mathrm{~Hz}, 3 \mathrm{H}, \mathrm{OCH}_{2} \mathrm{CH}_{3}\right), 2.37\left(\mathrm{~m}_{\mathrm{c}}, 2 \mathrm{H}, 3-\mathrm{H}\right), 3.23-3.75(\mathrm{~m}, 5 \mathrm{H}, 4$ $\left.\mathrm{H}, 5-\mathrm{H}, \mathrm{OCH}_{2} \mathrm{CH}_{3}\right), 4.97-5.27(\mathrm{~m}, 2 \mathrm{H}, 1-\mathrm{H}), 5.80\left(\mathrm{~m}_{\mathrm{c}}, 1 \mathrm{H}, 2-\mathrm{H}\right)$.

5-Bromo-4-methoxy-4-methyl-1-pentene (4b): Vinyl ether $1 \mathrm{~b}$ (720 mg, $10.0 \mathrm{mmol}), \mathrm{Br}_{2}(1.60 \mathrm{~g}, 10.0 \mathrm{mmol}), \mathrm{ZnCl}_{2}-\mathrm{Et}_{2} \mathrm{O}(2.5 \mathrm{ml}$, $4.84 \mathrm{mmol})$, and $3 \mathrm{a}(1.71 \mathrm{~g}, 15.0 \mathrm{mmol})$ gave $4 \mathrm{~b}(1.40 \mathrm{~g}, 73 \%)$ with b.p. $25-30^{\circ} \mathrm{C}$ (bath) $/ 0.4$ mbar (reaction time $15 \mathrm{~h}$ ). - ${ }^{1} \mathrm{H}$ NMR $\left(\mathrm{CCl}_{4}\right): \delta=1.22$ (s, $\left.3 \mathrm{H}, \mathrm{CH}_{3}\right), 2.35$ (br. d, $\left.J=6 \mathrm{~Hz}, 2 \mathrm{H}, 3-\mathrm{H}\right), 3.21$ (s, 3H, $\left.\mathrm{OCH}_{3}\right), 3.27$ (s, 2H, 5-H), 4.93-5.22 (m, 2H, 1-H), 5.47-5.97 (m, $1 \mathrm{H}, 2-\mathrm{H})$.

5-Bromo-4-ethoxy-1-hexene (4c): The dibromo ether prepared from $\mathrm{Br}_{2}(1.12 \mathrm{~g}, 7.01 \mathrm{mmol})$ and $(E, Z)-1 \mathrm{c}(600 \mathrm{mg}, 7.00 \mathrm{mmol}, E-$ $1 \mathrm{c}: Z-1 \mathrm{c}=30: 70)$ reacted within $15 \mathrm{~h}$ with $3 \mathrm{a}(1.20 \mathrm{~g}, 10.5 \mathrm{mmol})$ in the presence of $\mathrm{ZnCl}_{2}-\mathrm{Et}_{2} \mathrm{O}(1.8 \mathrm{ml}, 3.46 \mathrm{mmol})$ to give $4 \mathrm{c}$ $(1.33 \mathrm{~g}, 92 \%)$ with b.p. $40-45^{\circ} \mathrm{C}$ (bath) $/ 4$ mbar. - ${ }^{1} \mathrm{H}$ NMR $\left(\mathrm{CDCl}_{3}\right): \delta=1.21\left(\mathrm{t}, J=7.0 \mathrm{~Hz}, 3 \mathrm{H}, \mathrm{OCH}_{2} \mathrm{CH}_{3}\right), 1.70$ (d, $J=$ $6.8 \mathrm{~Hz}, 3 \mathrm{H}, 6-\mathrm{H}$ ), 2.42 (br. $\mathrm{t}, J=6.2 \mathrm{~Hz}, 2 \mathrm{H}, 3-\mathrm{H}$ ), 3.41 (q, $J=$ $5.4 \mathrm{~Hz}, 1 \mathrm{H}, 4-\mathrm{H}), 3.63\left(\mathrm{~m}_{\mathrm{c}}, 2 \mathrm{H}, \mathrm{OCH}_{2} \mathrm{CH}_{3}\right), 4.14$ (qd, $J=6.8 /$ $5.4 \mathrm{~Hz}, 1 \mathrm{H}, 5-\mathrm{H}), 5.06-5.20(\mathrm{~m}, 2 \mathrm{H}, 1-\mathrm{H}), 5.85\left(\mathrm{~m}_{\mathrm{c}}, 1 \mathrm{H}, 2-\mathrm{H}\right)$. ${ }^{13} \mathrm{C} \mathrm{NMR}\left(\mathrm{CDCl}_{3}\right): \delta=15.48\left(\mathrm{q}, \mathrm{OCH}_{2} \mathrm{CH}_{3}\right), 21.35$ (q, C-6), 36.49 (t, C-3), 51.31 (d, C-5), 66.52 (t, $\mathrm{OCH}_{2} \mathrm{CH}_{3}$ ), 83.02 (d, C-4), 117.58 (t, C-1), 134.13 (d, C-2). - The ${ }^{13} \mathrm{C}-\mathrm{NMR}$ spectrum indicates a $95: 5$ ratio of diastereomers. - MS $(70 \mathrm{eV}): m / z(\%)=165,167(94,92)$ $\left[\mathrm{M}^{+}-\mathrm{C}_{3} \mathrm{H}_{5}\right], 137,139(84,82)\left[\mathrm{M}^{+}-\mathrm{C}_{3} \mathrm{H}_{4}-\mathrm{C}_{2} \mathrm{H}_{4}\right], 57(100)$ $\left[\mathrm{M}^{+}-\mathrm{C}_{3} \mathrm{H}_{5}-\mathrm{C}_{2} \mathrm{H}_{4}-\mathrm{HBr}\right]$.

5-Bromo-4-methoxy-4-phenyl-1-pentene (4d): $5.00 \mathrm{~g}$ of a $83: 17$ mixture of $1 \mathrm{~d}$ and acetophenone dimethyl acetal (total $37.0 \mathrm{mmol}$ ), bromine (5.92 $\mathrm{g}, 37.0 \mathrm{mmol}), \mathrm{ZnCl}_{2}-\mathrm{Et}_{2} \mathrm{O}(9.3 \mathrm{ml}, 17.9 \mathrm{mmol})$, and $3 \mathrm{a}(6.30 \mathrm{~g}, 55.1 \mathrm{mmol})$ gave $8.67 \mathrm{~g}(92 \%)$ of spectroscopically pure 
4d. $-{ }^{1} \mathrm{H}$ NMR $\left(\mathrm{CDCl}_{3}\right): \delta=2.74$ (br. d, $J=6.5 \mathrm{~Hz}, 2 \mathrm{H}, 3-\mathrm{H}$ ), $3.10\left(\mathrm{~s}, 3 \mathrm{H}, \mathrm{OCH}_{3}\right), 3.69(\mathrm{~s}, 2 \mathrm{H}, 5-\mathrm{H}), 4.93-5.28(\mathrm{~m}, 2 \mathrm{H}, 1-\mathrm{H})$, $5.29-5.82(\mathrm{~m}, 1 \mathrm{H}, 2-\mathrm{H}), 7.28\left(\mathrm{~m}_{\mathrm{c}}, 5 \mathrm{H}, \mathrm{Ph}\right)$.

\section{4e: See typical procedure.}

5-Bromo-4-ethoxy-5-methyl-1-hexene (4f) was prepared in $81 \%$ yield $(3.92 \mathrm{~g})$ from $1 \mathrm{f}(2.20 \mathrm{~g}, 22.0 \mathrm{mmol})$, bromine $(3.20 \mathrm{~g}, 20.0$ $\mathrm{mmol}), \mathrm{ZnCl}_{2}-\mathrm{Et}_{2} \mathrm{O}(5.0 \mathrm{ml}, 9.60 \mathrm{mmol})$, and $3 \mathrm{a}(2.51 \mathrm{~g}, 22.0$ mmol): b.p. $62-73^{\circ} \mathrm{C} / 2-5$ mbar. $-{ }^{1} \mathrm{H}$ NMR $\left(\mathrm{CDCl}_{3}\right)$ : $\delta=1.17$ (t, $\left.J=6.0 \mathrm{~Hz}, 3 \mathrm{H}, \mathrm{OCH}_{2} \mathrm{CH}_{3}\right), 1.72,1.77\left(2 \mathrm{~s}, 6 \mathrm{H}, 5-\mathrm{CH}_{3}, 6-\mathrm{H}\right)$, $1.03-2.83(\mathrm{~m}, 2 \mathrm{H}, 3-\mathrm{H}), 3.30\left(\mathrm{~m}_{\mathrm{c}}, 1 \mathrm{H}, 4-\mathrm{H}\right), 3.63(\mathrm{q}, J=6.0 \mathrm{~Hz}$, $\left.2 \mathrm{H}, \mathrm{OCH}_{2} \mathrm{CH}_{3}\right), 4.87-5.27(\mathrm{~m}, 2 \mathrm{H}, 1-\mathrm{H}), 5.60-6.20(\mathrm{~m}, 1 \mathrm{H}, 2-\mathrm{H})$.

5-Bromo-4-methoxy-2,4-dimethyl-1-pentene $(\mathbf{4 g})$ : Vinyl ether $\mathbf{1 b}$ $(1.52 \mathrm{~g}, 21.1 \mathrm{mmol})$, bromine $(3.07 \mathrm{~g}, 19.2 \mathrm{mmol}), \mathrm{ZnCl}_{2}-\mathrm{Et}_{2} \mathrm{O}(4.8$ $\mathrm{ml}, 9.22 \mathrm{mmol})$, and $3 \mathrm{~b}(2.70 \mathrm{~g}, 21.0 \mathrm{mmol})$ gave $3.77 \mathrm{~g}(86 \%)$ of 4g: B.p. $50-70^{\circ} \mathrm{C}$ (bath) $/ 1 \mathrm{mbar}\left(\right.$ ref. ${ }^{10)} 30-40^{\circ} \mathrm{C}$ (bath) $/ 0.02 \mathrm{mbar}$ ). - 'H NMR $\left(\mathrm{CDCl}_{3}\right): \delta=1.27$ (s, $\left.3 \mathrm{H}, 4-\mathrm{CH}_{3}\right), 1.80$ (br. s, 3H, 2$\mathrm{CH}_{3}$ ), 2.40 (br. s, $\left.2 \mathrm{H}, 3-\mathrm{H}\right), 3.23$ (s, $\left.3 \mathrm{H}, \mathrm{OCH}_{3}\right), 3.40$ (s, $2 \mathrm{H}, 5-\mathrm{H}$ ), $4.80,4.90\left(2 \mathrm{~m}_{\mathrm{c}}, 2 \mathrm{H}, 1-\mathrm{H}\right)$.

5-Bromo-4-methoxy-2-methyl-4-phenyl-1-pentene (4h): $1.47 \mathrm{~g}$ of a 83:17 mixture of $1 \mathrm{~d}$ and acetophenone dimethyl acetal (total 11.0 mmol), bromine $(1.60 \mathrm{~g}, 10.0 \mathrm{mmol}), \mathrm{ZnCl}_{2}-\mathrm{Et}_{2} \mathrm{O}(2.5 \mathrm{ml}, 4.84$ $\mathrm{mmol})$, and $3 \mathrm{~b}(1.41 \mathrm{~g}, 11.0 \mathrm{mmol})$ gave $4 \mathrm{~h}(2.86 \mathrm{~g}, 97 \%)$ as an orange, spectroscopically pure liquid. $-{ }^{1} \mathrm{H}$ NMR $\left(\mathrm{CCl}_{4}\right): \delta=1.36$ (s, 3 H, 2- $\mathrm{CH}_{3}$ ), 2.50, 2.75 (AB system, $J=13.5 \mathrm{~Hz}, 2 \mathrm{H}, 3-\mathrm{H}$ ), 3.08 (s, 3 H, $\mathrm{OCH}_{3}$ ), 3.72, 3.90 (AB system, $J=9.0 \mathrm{~Hz}, 2 \mathrm{H}, \mathrm{BrCH}_{2}$ ), 4.73 $\left(\mathrm{m}_{\mathrm{c}}, 2 \mathrm{H}, \mathrm{C}=\mathrm{CH}_{2}\right), 7.28\left(\mathrm{~m}_{\mathrm{c}}, 5 \mathrm{H}, \mathrm{Ph}\right)$.

2-Bromo-1-ethoxy-1-(2-methylallyl)cyclohexane (4i): Compound 1 e $(2.77 \mathrm{~g}, 22.0 \mathrm{mmol})$, bromine $(3.20 \mathrm{~g}, 20.0 \mathrm{mmol}), \mathrm{ZnCl}_{2}-\mathrm{Et}_{2} \mathrm{O}$ $(5.0 \mathrm{ml}, 9.6 \mathrm{mmol})$, and $3 \mathrm{~b}(2.82 \mathrm{~g}, 22.0 \mathrm{mmol})$ gave $3.93 \mathrm{~g}(68 \%)$ of crude 4i. - ' ${ }^{1} \mathrm{H}$ NMR $\left(\mathrm{CDCl}_{3}\right): \delta=1.05-2.68(\mathrm{~m}, 16 \mathrm{H}, 3-, 4-$, 5-, 6-H, $\left.\mathrm{OCH}_{2} \mathrm{CH}_{3}, \mathrm{CH}_{3},=\mathrm{CCH}_{2}\right), 3.53\left(\mathrm{~m}_{\mathrm{c}}, 2 \mathrm{H}, \mathrm{OCH}_{2} \mathrm{CH}_{3}\right), 4.26$ $\left(\mathrm{m}_{\mathrm{c}}, 1 \mathrm{H}, 2-\mathrm{H}\right), 4.92$ (br. $\mathrm{s}, 2 \mathrm{H}, \mathrm{C}=\mathrm{CH}_{2}$ ).

5-Bromo-4-ethoxy-2,5-dimethyl-1-hexene (4j) was obtained in $92 \%$ yield $(1.19 \mathrm{~g})$ from $1 \mathrm{f}(550 \mathrm{mg}, 5.49 \mathrm{mmol})$, bromine $(800 \mathrm{mg}$, $5.00 \mathrm{mmol}$ ), $\mathrm{ZnCl}_{2}-\mathrm{Et}_{2} \mathrm{O}(1.3 \mathrm{ml}, 2.52 \mathrm{mmol}$ ), and $3 \mathrm{~b}$ (705 $\mathrm{mg}, 5.50$ mmol). - ${ }^{1} \mathrm{H}$ NMR $\left(\mathrm{CDCl}_{3}\right): \delta=1.18(\mathrm{t}, J=6 \mathrm{~Hz}, 3 \mathrm{H}$, $\left.\mathrm{OCH}_{2} \mathrm{CH}_{3}\right), 1.71,1.78,1.85\left[3 \mathrm{~s}, 9 \mathrm{H}, \mathrm{BrC}\left(\mathrm{CH}_{3}\right)_{2}, 2-\mathrm{CH}_{3}\right], 2.02-2.70$ (m, 2H, 3-H), $3.31-3.84$ (m, 3H, $\mathrm{OCH}_{2} \mathrm{CH}_{3}, 4-\mathrm{H}$ ), 4.83 (br. s, $2 \mathrm{H}$, $\mathrm{C}=\mathrm{CH}_{2}$ ).

5-Bromo-4-ethoxy-2,3,3-trimethyl-1-pentene (4k): Vinyl ether $1 \mathrm{a}$ $(1.59 \mathrm{~g}, 22.0 \mathrm{mmol})$, bromine $(3.20 \mathrm{~g}, 20.0 \mathrm{mmol}), \mathrm{ZnCl}_{2}-\mathrm{Et}_{2} \mathrm{O}$ $(5.0 \mathrm{ml}, 9.60 \mathrm{mmol})$, and $3 \mathrm{c}(4.37 \mathrm{~g}, 22.0 \mathrm{mmol})$ gave $8.52 \mathrm{~g}$ of a mixture of $4 \mathbf{k}$ and of hexaethyldisiloxane which was not separated but subjected to treatment with sodium (see below). The spectral data of $4 \mathbf{k}$ were taken from this mixture. $-{ }^{1} \mathrm{H} \mathrm{NMR}\left(\mathrm{CDCl}_{3}\right): \delta=$ $1.03,1.10\left(2 \mathrm{~s}, 6 \mathrm{H}, 3-\mathrm{CH}_{3}\right), 1.20\left(\mathrm{t}, J=6.0 \mathrm{~Hz}, 3 \mathrm{H}, \mathrm{OCH}_{2} \mathrm{CH}_{3}\right)$, $1.77\left(\mathrm{~s}, 3 \mathrm{H}, 2-\mathrm{CH}_{3}\right), 3.10-4.06\left(\mathrm{~m}, 5 \mathrm{H}, \mathrm{OCH}_{2} \mathrm{CH}_{3}, 4-, 5-\mathrm{H}\right), 4.82$ (br. s, $2 \mathrm{H}, \mathrm{C}=\mathrm{CH}_{2}$ ).

1-(2-Bromo-1-ethoxypropyl)-2-methylenecyclohexane (41): Compound $1 \mathrm{c}(E, Z$ mixture, $1.57 \mathrm{~g}, 18.2 \mathrm{mmol})$, bromine $(2.64 \mathrm{~g}, 16.5$ $\mathrm{mmol}), \mathrm{ZnCl}_{2}-\mathrm{Et}_{2} \mathrm{O}(3.6 \mathrm{ml}, 6.90 \mathrm{mmol})$, and $3 \mathrm{~d}(3.10 \mathrm{~g}, 18.4$ mmol) gave $4.70 \mathrm{~g}(99 \%)$ of 41 . - ${ }^{1} \mathrm{H}$ NMR $\left(\mathrm{CDCl}_{3}\right): \delta=$ $1.08-2.73\left(\mathrm{~m}, 14 \mathrm{H}, 2 \mathrm{CH}_{3}\right.$ and cyclohexane $\left.\mathrm{H}\right), 3.33-4.56(\mathrm{~m}, 5 \mathrm{H}$, $\left.\mathrm{OCH}_{2} \mathrm{CH}_{3}, \mathrm{BrCH}, \mathrm{OCH}, \mathrm{CH}\right), 4.60-4.93\left(\mathrm{~m}, 2 \mathrm{H}, \mathrm{C}=\mathrm{CH}_{2}\right)$. On the basis of ' $\mathrm{H}$-NMR spectral data it could not be decided whether this was a mixture of two diastereoisomers or a single isomer.

2-Bromo-1-(2-cyclopenten-1-yl)-1-methoxy-1-phenylethane (4m) was obtained in $92 \%$ yield ( $2.83 \mathrm{~g})$ from a $83: 17$ mixture of $1 \mathbf{d}$ and acetophenone dimethyl acetal $(1.47 \mathrm{~g}$, total $11.0 \mathrm{mmol})$, bromine $(1.60 \mathrm{~g}, 10.0 \mathrm{mmol}), \mathrm{ZnCl}_{2}-\mathrm{Et}_{2} \mathrm{O}(2.5 \mathrm{ml}, 4.80 \mathrm{mmol})$, and $3 \mathbf{e}$
$(1.54 \mathrm{~g}, 11.0 \mathrm{mmol}) .-{ }^{1} \mathrm{H}$ NMR $\left(\mathrm{CCl}_{4}\right): \delta=1.66-1.93(\mathrm{~m}, 4 \mathrm{H}$, $\left.\mathrm{CH}_{2} \mathrm{CH}_{2}\right), 3.29\left(\mathrm{~s}, 3 \mathrm{H}, \mathrm{OCH}_{3}\right), 3.38-4.06(\mathrm{~m}, 3 \mathrm{H}, \mathrm{CH}-\mathrm{CH}=$, $\left.\mathrm{BrCH}_{2}\right), 5.68\left(\mathrm{~m}_{\mathrm{c}}, 2 \mathrm{H}\right.$, vinyl $\left.\mathrm{H}\right), 7.21\left(\mathrm{~m}_{\mathrm{c}}, 5 \mathrm{H}, \mathrm{Ph}\right)$.

2-Bromo-1-(2-cyclopenten-1-yl)-2-ethoxycyclohexane (4n): Enol ether $1 \mathrm{e}(2.77 \mathrm{~g}, 22.0 \mathrm{mmol})$, bromine $(3.20 \mathrm{~g}, 20.0 \mathrm{mmol})$, $\mathrm{ZnCl}_{2}-\mathrm{Et}_{2} \mathrm{O}(5.0 \mathrm{ml}, 9.60 \mathrm{mmol})$, and $3 \mathrm{e}(3.08 \mathrm{~g}, 22.0 \mathrm{mmol})$ gave $5.29 \mathrm{~g}(88 \%)$ of $4 \mathrm{n} .-{ }^{1} \mathrm{H} \mathrm{NMR}\left(\mathrm{CDCl}_{3}\right): \delta=0.99-2.57(\mathrm{~m}, 15 \mathrm{H}$, $\left.6 \mathrm{CH}_{2}, \mathrm{OCH}_{2} \mathrm{CH}_{3}\right), 3.28-4.04\left(\mathrm{~m}, 3 \mathrm{H}, \mathrm{OCH}_{2} \mathrm{CH}_{3}, \mathrm{CH}-\mathrm{CH}=\right.$ ), $4.18\left(\mathrm{~m}_{\mathrm{c}}, 1 \mathrm{H}, \mathrm{BrCH}\right), 5.78\left(\mathrm{~m}_{\mathrm{c}}, 2 \mathrm{H}\right.$, vinyl $\left.\mathrm{H}\right)$.

2-Bromo-1-(2-cyclopenten-1-yl)-1-ethoxy-2-methylpropane (40) was synthesized in $88 \%$ yield $(1.20 \mathrm{~g})$ from enol ether $1 \mathrm{f}(550 \mathrm{mg}$, $5.49 \mathrm{mmol})$, bromine $(800 \mathrm{mg}, 5.00 \mathrm{mmol}), \mathrm{ZnCl}_{2}-\mathrm{Et}_{2} \mathrm{O}(1.3 \mathrm{ml}$, $2.52 \mathrm{mmol})$, and $3 \mathrm{e}(770 \mathrm{mg}, 5.50 \mathrm{mmol}) .-{ }^{1} \mathrm{H} \mathrm{NMR}\left(\mathrm{CDCl}_{3}\right): \delta=$ $0.95-1.32\left(\mathrm{~m}, 4 \mathrm{H}, 5^{\prime}-\mathrm{H}_{\mathrm{A}}, \mathrm{OCH}_{2} \mathrm{CH}_{3}\right), 1.52-2.50\left[\mathrm{~m}, 9 \mathrm{H}, 4^{\prime}-\mathrm{H}, 5^{\prime}-\right.$ $\left.\mathrm{H}_{\mathrm{B}}, \mathrm{C}\left(\mathrm{CH}_{3}\right)_{2}\right], 3.11-3.75\left(\mathrm{~m}, 4 \mathrm{H}, 1^{\prime}-\mathrm{H}, \mathrm{CHOCH} \mathrm{CH}_{3}\right), 5.69\left(\mathrm{~m}_{\mathrm{c}}\right.$, $2 \mathrm{H}$, vinyl $\mathrm{H})$.

\section{Synthesis of the 1,4-Dienes}

Elimination with Zinc/Ethanol (Procedure A): Crude $\mathbf{4 h}$, obtained from $11.0 \mathrm{mmol}$ of $\mathbf{1 d}$ and $\mathbf{3 b}$, was dissolved in absolute ethanol $(28 \mathrm{ml})$. After addition of zinc powder $(6.50 \mathrm{~g}, 100 \mathrm{mmol})$, the mixture was heated at reflux for $15 \mathrm{~h}$. Pentane $(40 \mathrm{ml})$ was added, the solvent mixture was decanted, and the remaining zinc powder was washed with four $20-\mathrm{ml}$ portions of pentane. The combined solvents were successively washed with $10 \%$ aqueous $\mathrm{NH}_{4} \mathrm{Cl}$ solution $(40 \mathrm{ml})$ and with four $20-\mathrm{ml}$ portions of water. The solvent was evaporated, and the residue was distilled to give $1.30 \mathrm{~g}(75 \%)$ of 5 h with b.p. $100^{\circ} \mathrm{C}$ (bath) $/ 10-20$ mbar (ref. ${ }^{15)} 90-92^{\circ} \mathrm{C} / 16$ mbar). For spectral data see below.

Elimination with Sodium in Diethyl Ether (Procedure B): Dry diethyl ether $(40-50 \mathrm{ml})$ and sodium wire $(3.79 \mathrm{~g}, 165 \mathrm{mmol})$ were placed into a $250-\mathrm{ml}$ two-necked flask under nitrogen. Crude $4 \mathbf{i}$, prepared from $22.0 \mathrm{mmol}$ of $1 \mathrm{e}$ and $3 \mathrm{~b}$, was added, and the mixture was heated under reflux for $4 \mathrm{~h}$. The solvent was decanted, and the solid residue was washed with pentane $(20 \mathrm{ml}, 10 \mathrm{ml}$, and $5 \mathrm{ml})$. The solutions were combined, washed with two $40-\mathrm{ml}$ portions of water (caution: small pieces of $\mathrm{Na}$ may be in the solution!), and dried with $\mathrm{Na}_{2} \mathrm{SO}_{4}$. After careful evaporation of the solvents, the residue was distilled to give $2.20 \mathrm{~g}(73 \%)$ of $5 \mathrm{i}$ with b.p. $68^{\circ} \mathrm{C}$ (bath)/ 5-20 mbar. For spectral data see below.

2-Phenyl-1,4-pentadiene (5d): Compound 4d, obtained from 22.0 mmol $1 \mathrm{~d}$ and $3 \mathrm{a}$, gave $2.50 \mathrm{~g} \mathrm{(79 \% )}$ of $5 \mathrm{~d}$ according to procedure A. B.p. $80^{\circ} \mathrm{C}$ (bath) $/ 22-30$ mbar (ref. ${ }^{15)} 82-84^{\circ} \mathrm{C} / 16$ mbar). $-{ }^{1} \mathrm{H}$ NMR $\left(\mathrm{CDCl}_{3}\right): \delta=3.22$ (br. d, $\left.J=6.7 \mathrm{~Hz}, 2 \mathrm{H}, 3-\mathrm{H}\right), 4.93-5.21$ $\left(\mathrm{m}, 3 \mathrm{H}, 1-\mathrm{H}_{\mathrm{A}}, 5-\mathrm{H}\right), 5.38\left(\mathrm{~s}, 1 \mathrm{H}, 1-\mathrm{H}_{\mathrm{B}}\right), 5.88\left(\mathrm{~m}_{\mathrm{c}}, 1 \mathrm{H}, 4-\mathrm{H}\right), 7.21-7.45$ $(\mathrm{m}, 5 \mathrm{H}, \mathrm{Ph}) .-{ }^{13} \mathrm{C}$ NMR $\left(\mathrm{CDCl}_{3}\right): \delta=39.49(\mathrm{t}, \mathrm{C}-3), 113.10(\mathrm{t}, \mathrm{C}-$ 1), 116.45 (t, C-5), 125.95 (d, $\left.C_{o}\right), 127.41\left(\mathrm{~d}, \mathrm{C}_{p}\right), 128.22\left(\mathrm{~d}, \mathrm{C}_{m}\right), 136.14$ (d, C-4), $140.88\left(\mathrm{~s}, \mathrm{C}_{i}\right), 146.25$ (s, C-2). - MS $(70 \mathrm{eV}): m / z(\%)=$ $144(100)\left[\mathrm{M}^{+}\right], 129(73)\left[\mathrm{M}^{+}-\mathrm{CH}_{3}\right], 115(10)\left[\mathrm{M}^{+}-\mathrm{C}_{2} \mathrm{H}_{5}\right]$, $103(57)\left[\mathrm{M}^{+}-\mathrm{C}_{3} \mathrm{H}_{5}\right], 77(16)\left[\mathrm{Ph}^{+}\right]$.

\section{$\mathrm{C}_{11} \mathrm{H}_{12}$ Calcd. 144.0939 Found 144.0932 (MS)}

1-Allyl-1-cyclohexene (5e): Procedure A was used to prepare $272 \mathrm{mg} \mathrm{(40 \%} \mathrm{with} \mathrm{respect} \mathrm{to} 1$ e) from crude 4 e. B.p. $55^{\circ} \mathrm{C}$ (bath)/ 5-20 mbar (ref. $\left.{ }^{16)} 154-158^{\circ} \mathrm{C}\right)$ - - 'H NMR $\left(\mathrm{CDCl}_{3}\right): \delta=$ $1.54-1.64(\mathrm{~m}, 4 \mathrm{H}, 4-, 5-\mathrm{H}), 1.89-2.01(\mathrm{~m}, 4 \mathrm{H}, 3-, 6-\mathrm{H}), 2.67$ (d, $J=6.9 \mathrm{~Hz}, 2 \mathrm{H}$, allyl $\left.\mathrm{CH}_{2}\right), 4.97-5.08\left(\mathrm{~m}, 2 \mathrm{H}, \mathrm{CH}=\mathrm{CH}_{2}\right), 5.44$ $\left(\mathrm{m}_{\mathrm{c}}, 1 \mathrm{H}, 2-\mathrm{H}\right), 5.70-5.91\left(\mathrm{~m}, 1 \mathrm{H}, \mathrm{CH}=\mathrm{CH}_{2}\right) .-{ }^{13} \mathrm{C} \mathrm{NMR}\left(\mathrm{CDCl}_{3}\right)$ : $\delta=22.50,22.97,25.31,28.34(4 \mathrm{t}, \mathrm{C}-3,-4,-5,-6), 42.57\left(\mathrm{t}, \mathrm{CH}_{2} \mathrm{CH}\right)$, 115.44 (t, $\mathrm{CH}=\mathrm{CH}_{2}$ ), 121.87 (d, C-2), 136.30 (s, C-1), 136.97 (d, $\left.\mathrm{CH}=\mathrm{CH}_{2}\right) .-\mathrm{MS}(70 \mathrm{eV}): m / z(\%)=122(28)\left[\mathrm{M}^{+}\right], 107(14)$ $\left[\mathrm{M}^{+}-\mathrm{CH}_{3}\right], 93(16)\left[\mathrm{M}^{+}-\mathrm{C}_{2} \mathrm{H}_{5}\right], 81(100)\left[\mathrm{M}^{+}-\mathrm{C}_{3} \mathrm{H}_{5}\right]$. $\mathrm{C}_{9} \mathrm{H}_{14}$ Calcd. 122.1096 Found 122.1091 (MS) 
2-Methyl-4-phenyl-1,4-pentadiene (5h) was synthesized according to procedure A (yield 75\%, details see above) and procedure $B$ (yield $2.26 \mathrm{~g}=65 \%$ with respect to $1 \mathrm{~d})$. $-{ }^{1} \mathrm{H} \mathrm{NMR}\left(\mathrm{CDCl}_{3}\right): \delta=1.72$ (s, $\left.3 \mathrm{H}, \mathrm{CH}_{3}\right), 3.21(\mathrm{~s}, 2 \mathrm{H}, 3-\mathrm{H}), 4.77,4.81\left(2 \mathrm{~m}_{\mathrm{c}}, 2 \mathrm{H}, 1-\mathrm{H}\right), 5.11$ (d, $J=1.4 \mathrm{~Hz}, 5-\mathrm{H}), 5.43(\mathrm{~d}, J=1.5 \mathrm{~Hz}, 1 \mathrm{H}, 5-\mathrm{H}), 7.27-7.46(\mathrm{~m}$, $5 \mathrm{H}, \mathrm{Ph}) .-{ }^{13} \mathrm{C} \mathrm{NMR}\left(\mathrm{CDCl}_{3}\right): \delta=22.28\left(\mathrm{q}, \mathrm{CH}_{3}\right), 44.00(\mathrm{t}, \mathrm{C}-3)$, 112.58, 114.27 (2 t, C-1, -5), 126.07 (d, $\left.C_{o}\right), 127.34$ (d, $\left.C_{p}\right), 128.15$ (d, $\left.\mathrm{C}_{m}\right), 140.96,143.39,145.59$ (3 s, $\left.\mathrm{C}_{i}, \mathrm{C}-2,-4\right) . \quad$ MS (70 eV): $m / z$ $(\%)=158(14)\left[\mathrm{M}^{+}\right], 143(100)\left[\mathrm{M}^{+}-\mathrm{CH}_{3}\right], 128(30)\left[\mathrm{M}^{+}-\right.$ $\left.2 \mathrm{CH}_{3}\right], 103(33)\left[\mathrm{M}^{+}-\mathrm{C}_{4} \mathrm{H}_{7}\right], 77(22)\left[\mathrm{Ph}^{+}\right]$.

\section{$\mathrm{C}_{12} \mathrm{H}_{14}$ Calcd. 158.1096 Found 158.1095 (MS)}

1-(2-Methylallyl)-1-cyclohexene (5i) was prepared following procedure A (yield $459 \mathrm{mg}=61 \%$ with respect to $1 \mathrm{e}$ ) and $\mathrm{B}$ (yield $74 \%$, details see above). - ${ }^{1} \mathrm{H}$ NMR $\left(\mathrm{CDCl}_{3}\right): \delta=1.50-1.64(\mathrm{~m}$, $\left.7 \mathrm{H}, \mathrm{CH}_{3}, 4-, 5-\mathrm{H}\right), 1.81-2.10(\mathrm{~m}, 4 \mathrm{H}, 3-, 6-\mathrm{H}), 2.63$ (s, 2H, allyl $\left.\mathrm{CH}_{2}\right), 4.70,4.75\left(2 \mathrm{~m}_{\mathrm{c}}, 2 \mathrm{H}, \mathrm{C}=\mathrm{CH}_{2}\right), 5.46\left(\mathrm{~m}_{\mathrm{c}}, 1 \mathrm{H}, 2-\mathrm{H}\right) .-{ }^{13} \mathrm{C}$ NMR $\left(\mathrm{CDCl}_{3}\right): \delta=21.84\left(\mathrm{q}, \mathrm{CH}_{3}\right), 22.49,23.00,25.35,27.70$ (4 t, $\mathrm{C}-3,-4,-5,-6), 47.14$ (t, $\left.\mathrm{CH}_{2}\right), 111.28$ (t, $\left.\mathrm{C}=\mathrm{CH}_{2}\right), 123.00$ (d, C-2), 135.51 (s, C-1), 144.28 (s, $\left.C=\mathrm{CH}_{2}\right)$. $-\mathrm{MS}(70 \mathrm{eV}): m / z(\%)=136$ (30) $\left[\mathrm{M}^{+}\right], 121$ (64) $\left[\mathrm{M}^{+}-\mathrm{CH}_{3}\right], 107$ (21) $\left[\mathrm{M}^{+}-\mathrm{C}_{2} \mathrm{H}_{5}\right], 93$ (49) $\left[\mathrm{M}^{+}-\mathrm{C}_{3} \mathrm{H}_{7}\right], 81(100)\left[\mathrm{M}^{+}-\mathrm{C}_{4} \mathrm{H}_{7}\right]$.

$\mathrm{C}_{10} \mathrm{H}_{16} \quad$ Calcd. 136.1252 Found 136.1257 (MS)

2,5-Dimethyl-1,4-hexadiene (5j) has been synthesized according to procedure A (yield $122 \mathrm{mg}=20 \%$ with respect to $1 \mathrm{f}$ ) and procedure B $\left(1.52 \mathrm{~g}=72 \%\right.$ with respect to 1 f). B.p. $89^{\circ} \mathrm{C}$ (bath)/ 150-190 mbar (ref. ${ }^{17)}$ does not report the b.p.). - ${ }^{1} \mathrm{H}$ NMR $\left(\mathrm{CDCl}_{3}\right): \delta=1.63,1.72$ [2 br. s, $\left.9 \mathrm{H}, 2-\mathrm{CH}_{3}, \mathrm{C}\left(\mathrm{CH}_{3}\right)_{2}\right], 2.68$ (br. d, $J=7.1 \mathrm{~Hz}, 2 \mathrm{H}, 3-\mathrm{H}), 4.69\left(\mathrm{~m}_{\mathrm{c}}, 2 \mathrm{H}, 1-\mathrm{H}\right), 5.19\left(\mathrm{~m}_{\mathrm{c}}, 1 \mathrm{H}, 4-\mathrm{H}\right)$. ${ }^{13} \mathrm{C}$ NMR $\left(\mathrm{CDCl}_{3}\right): \delta=17.62,22.55,25.77\left(3 \mathrm{q}, 3 \mathrm{CH}_{3}\right), 36.59$ (t, C-3), 109.71 (t, C-1), 121.94 (d, C-4), 132.92 (s, C-5), 145.46 (s, C-2). - MS $(70 \mathrm{eV}): m / z(\%)=110(52)\left[\mathrm{M}^{+}\right], 95(100)\left[\mathrm{M}^{+}-\mathrm{CH}_{3}\right]$, 67 (47) $\left[\mathrm{M}^{+}-\mathrm{C}_{3} \mathrm{H}_{7}\right]$.

\section{$\mathrm{C}_{8} \mathrm{H}_{14}$ Calcd. 110.1096 Found 110.1098 (MS)}

2,3,3-Trimethyl-1,4-pentadiene (5k): The mixture of $4 \mathbf{k}$ and hexaethyldisiloxane described above was treated with $\mathrm{Na}$ (procedure $\mathrm{B}$ ) to give $1.50 \mathrm{~g}(62 \%$ with respect to $1 \mathrm{a})$ of 5 k. B.p. ${ }^{18)} 102{ }^{\circ} \mathrm{C} .-{ }^{1} \mathrm{H}$ NMR $\left(\mathrm{CDCl}_{3}\right): \delta=1.15\left[\mathrm{~s}, 6 \mathrm{H}, \mathrm{C}\left(\mathrm{CH}_{3}\right)_{2}\right], 1.70\left(\mathrm{~m}_{\mathrm{c}}, 3 \mathrm{H}, 2-\mathrm{CH}_{3}\right)$, $4.74-4.82(\mathrm{~m}, 2 \mathrm{H}, 5-\mathrm{H}), 4.93-5.03(\mathrm{~m}, 2 \mathrm{H}, 1-\mathrm{H}), 5.82\left(\mathrm{~m}_{\mathrm{c}}, 1 \mathrm{H}, 4-\right.$ $\mathrm{H}) .-{ }^{13} \mathrm{C} \mathrm{NMR}\left(\mathrm{CDCl}_{3}\right): \delta=19.74\left(\mathrm{q}, 2-\mathrm{CH}_{3}\right), 26.01\left[\mathrm{q}, \mathrm{C}\left(\mathrm{CH}_{3}\right)_{2}\right]$, 42.18 (s, C-3), 109.30, 110.87 (2 t, C-1, -5), 147.14 (d, C-4), 151.61 (s, C-2). - MS (70 eV): $m / z(\%)=110(20)\left[\mathrm{M}^{+}\right], 95(100)\left[\mathrm{M}^{+}-\right.$ $\left.\mathrm{CH}_{3}\right], 67$ (44) $\left[\mathrm{M}^{+}-\mathrm{C}_{3} \mathrm{H}_{7}\right], 41$ (47) $\left[\mathrm{C}_{3} \mathrm{H}_{5}^{+}\right]$.

$\mathrm{C}_{8} \mathrm{H}_{14}$ Calcd. 110.1096 Found 110.1098 (MS)

1-Methylene-2-((E,Z)-1-propenyl) cyclohexane (51): Treatment of crude $41(4.70 \mathrm{~g})$ with $\mathrm{Na}$ according to procedure $\mathrm{B}$ gave $1.60 \mathrm{~g}$ $(64 \%$ with respect to $1 \mathrm{c})$ of $5 \mathrm{l}$ as a mixture of $(E)$ - and $(Z)-5 \mathrm{l}$. B.p. $90^{\circ} \mathrm{C}$ (bath) $/ 16-90$ mbar. - ${ }^{1} \mathrm{H}$ NMR $\left(\mathrm{CDCl}_{3}\right): \delta=1.20-1.90$ (m, 9H, 3-, 4-, 5-H, $\left.\mathrm{CH}_{3}\right), 2.02-2.22,2.32-2.50(2 \mathrm{~m}, 2 \mathrm{H}, 6-\mathrm{H})$, $2.66-2.80,2.98-3.12(2 \mathrm{~m}, 1 \mathrm{H}, 2-\mathrm{H}), 4.64-4.80\left(\mathrm{~m}, 2 \mathrm{H}, \mathrm{C}=\mathrm{CH}_{2}\right)$, $5.40-5.72(\mathrm{~m}, 2 \mathrm{H}, \mathrm{CH}=\mathrm{CH}) .-{ }^{13} \mathrm{C} \mathrm{NMR}\left(\mathrm{CDCl}_{3}\right): \delta=12.96(\mathrm{q}$, $\mathrm{CH}_{3}, \mathrm{Z}$ ), 18.08 (q, $\left.\mathrm{CH}_{3}, E\right), 25.07,25.42,28.16,28.27,34.45,34.79$, $35.29,35.54$ ( $8 \mathrm{t}, \mathrm{C}-3,-4,-5,-6, E, Z), 41.14,46.59$ (2 d, C-2, E.Z), $106.10, \quad 106.34 \quad\left(2 \mathrm{t}, \quad \mathrm{C}=\mathrm{CH}_{2}, \quad E, Z\right), \quad 123.95, \quad 124.86 \quad(2 \mathrm{~d}$, $\left.\mathrm{CH}_{3} \mathrm{CH}=\mathrm{CH}, E, Z\right), 132.77,133.69\left(2 \mathrm{~d}, \mathrm{CH}_{3} \mathrm{CH}=C \mathrm{H}, E, Z\right), 151.00$, 152.54 (2 s. C-1, E,Z). - MS (70 eV): $m / z(\%)=136(64)\left[\mathrm{M}^{+}\right]$, $121(60)\left[\mathrm{M}^{+}-\mathrm{CH}_{3}\right], 107(50)\left[\mathrm{M}^{+}-\mathrm{C}_{2} \mathrm{H}_{5}\right], 93(76)\left[\mathrm{M}^{+}-\right.$ $\left.\mathrm{C}_{3} \mathrm{H}_{7}\right], 79(100)\left[\mathrm{M}^{+}-\mathrm{C}_{2} \mathrm{H}_{5}-\mathrm{C}_{2} \mathrm{H}_{4}\right]$.

\section{$\mathrm{C}_{10} \mathrm{H}_{16} \quad$ Calcd. 136.1252 Found 136.1256 (MS)}

3-(1-Phenylvinyl)-1-cyclopentene $(5 \mathrm{~m})$ : $1.41 \mathrm{~g}$ of compound $5 \mathrm{~m}$ was obtained from crude $4 \mathrm{~m}$ and $\mathrm{Zn}$ (procedure A). Yield: $75 \%$ (with respect to 1d). B.p. $87-110^{\circ} \mathrm{C}$ (bath)/2-4 mbar. - 'H NMR
$\left(\mathrm{CDCl}_{3}\right): \delta=1.46-1.68\left(\mathrm{~m}, 1 \mathrm{H}, 4-\mathrm{H}_{\mathrm{A}}\right), 2.10-2.48\left(\mathrm{~m}, 3 \mathrm{H}, 4-\mathrm{H}_{\mathrm{B}}\right.$ 5-H), $3.85\left(\mathrm{~m}_{\mathrm{c}}, 1 \mathrm{H}, 3-\mathrm{H}\right), 5.03,5.25\left(2 \mathrm{~m}_{\mathrm{c}}, 2 \mathrm{H}, \mathrm{C}=\mathrm{CH}_{2}\right), 5.70-5.92$ (m, 2H, 1-, 2-H), 7.25-7.47 (m, 5H, Ph). $-{ }^{13} \mathrm{C} \mathrm{NMR}\left(\mathrm{CDCl}_{3}\right)$ : $\delta=31.14,32.00$ (2 t, C-4, -5), 49.77 (d, C-3), 110.81 (t, C=CH ), 126.38 (d, C C $\left._{o}\right), 127.19$ (d, C $\left.)_{p}\right) 128.17$ (d, C $C_{m}$ ), 131.90, 133.29 (2 d, $\mathrm{C}-1,-2), 141.95\left(\mathrm{~s}, \mathrm{C}_{i}\right), 152.24\left(\mathrm{~s}, \mathrm{C}=\mathrm{CH}_{2}\right) .-\mathrm{MS}(70 \mathrm{eV}): \mathrm{m} / z(\%)=$ $170(100)\left[\mathrm{M}^{+}\right], 155(57)\left[\mathrm{M}^{+}-\mathrm{CH}_{3}\right], 103(75)\left[\mathrm{M}^{+}-\right.$cyclopentenyl], $77(52)\left[\mathrm{Ph}^{+}\right], 67(40)$ [cyclopentenyl $\left.{ }^{+}\right]$.

\section{$\mathrm{C}_{13} \mathrm{H}_{14}$ Calcd. 170.1096 Found 170.1086 (MS)}

1-(2-Cyclopenten-1-yl)-1-cyclohexene (5n): Procedure B yielded $1.77 \mathrm{~g}\left(54 \%\right.$ with respect to 1 e) of $5 \mathrm{n}$ from crude 4 n. B.p. $100^{\circ} \mathrm{C} /$ 16-20 mbar (ref. ${ }^{19)}$ does not report the b.p.). $-{ }^{1} \mathrm{H} \mathrm{NMR}\left(\mathrm{CDCl}_{3}\right)$ : $\delta=1.50-1.78(\mathrm{~m}, 5 \mathrm{H}), 1.89-2.22(\mathrm{~m}, 5 \mathrm{H}), 2.37\left(\mathrm{~m}_{\mathrm{c}}, 2 \mathrm{H}\right), 3.27\left(\mathrm{~m}_{\mathrm{c}}\right.$, $1 \mathrm{H}, 3-\mathrm{H}), 5.43\left(\mathrm{~m}_{\mathrm{c}}, 1 \mathrm{H}\right), 5.64-5.90(\mathrm{~m}, 2 \mathrm{H}, 1-, 2-\mathrm{H}) .-{ }^{13} \mathrm{C}$ NMR $\left(\mathrm{CDCl}_{3}\right): \delta=22.73(\mathrm{t}), 23.09(\mathrm{t}), 25.24(\mathrm{t}), 26.40(\mathrm{t}), 29.30(\mathrm{t}), 32.38$ (t), 52.93 (d), 119.73 (d), 131.23 (d), 133.65 (d), 140.97 (s). - MS $(70 \mathrm{eV}): m / z(\%)=148(86)\left[\mathrm{M}^{+}\right], 133(15)\left[\mathrm{M}^{+}-\mathrm{CH}_{3}\right], 119$ (36) $\left[\mathrm{M}^{+}-\mathrm{C}_{2} \mathrm{H}_{5}\right], 105(33)\left[\mathrm{M}^{+}-\mathrm{C}_{3} \mathrm{H}_{7}\right], 91(65)\left[\mathrm{M}^{+}-\mathrm{C}_{4} \mathrm{H}_{9}\right], 80$ (100), 77 (25), 67 (53) [cyclopentenyl ${ }^{+}$].

\section{$\mathrm{C}_{11} \mathrm{H}_{16}$ Calcd. 148.1252 Found 148.1258 (MS)}

3-(2-Methyl-1-propenyl)-1-cyclopentene (5o) was prepared according to procedure A (yield $200 \mathrm{mg}=30 \%$ with respect to $1 \mathrm{f}$ ) and procedure $\mathrm{B}(1.80 \mathrm{~g}=67 \%$ with respect to $1 \mathrm{f})$. B.p. $73^{\circ} \mathrm{C}$ (bath)/30-45 mbar (ref. ${ }^{(9)}$ does not report the b.p.). - ${ }^{1} \mathrm{H}$ NMR $\left(\mathrm{CDCl}_{3}\right): \delta=1.36-1.55\left(\mathrm{~m}, 1 \mathrm{H}, 4-\mathrm{H}_{\mathrm{A}}\right), 1.67,1.69$ [2 br. s, $6 \mathrm{H}$, $\left.\mathrm{C}\left(\mathrm{CH}_{3}\right)_{2}\right], 2.02-2.40\left(\mathrm{~m}, 3 \mathrm{H}, 4-\mathrm{H}_{\mathrm{B}}, 5-\mathrm{H}\right), 3.49\left(\mathrm{~m}_{\mathrm{c}}, 1 \mathrm{H}, 3-\mathrm{H}\right), 5.00$ (br. d, $J=9.1 \mathrm{~Hz}, 1 \mathrm{H}, \mathrm{C}=\mathrm{CH}), 5.50-5.80(\mathrm{~m}, 2 \mathrm{H}, 1-, 2-\mathrm{H})$. ${ }^{13} \mathrm{C}$ NMR $\left(\mathrm{CDCl}_{3}\right): \delta=17.93,25.73\left[2 \mathrm{q}, \mathrm{C}\left(\mathrm{CH}_{3}\right)_{2}\right], 31.19,32.22$ (2 t, C-4, -5), 44.25 (d, C-3), 129.13, 130.45 (2 d, C-1, -2), 130.60 [s, $\left.\left(\mathrm{CH}_{3}\right)_{2} \mathrm{C}=\mathrm{CH}\right], 134.72\left[\mathrm{~d},\left(\mathrm{CH}_{3}\right) \mathrm{C}=\mathrm{CH}\right] .-\mathrm{MS}(70 \mathrm{eV}): \mathrm{m} / \mathrm{z}$ $(\%)=122(50)\left[\mathrm{M}^{+}\right], 107(100)\left[\mathrm{M}^{+}-\mathrm{CH}_{3}\right], 79(64)\left[\mathrm{M}^{+}-\right.$ $\mathrm{C}_{3} \mathrm{H}_{7}$ ], 67 (17) [cyclopentenyl ${ }^{+}$].

\section{$\mathrm{C}_{9} \mathrm{H}_{14}$ Calcd. 122.1096 Found 122.1098 (MS)}

The reaction with $\mathrm{Zn}$ in ethanol gave compounds 6 and 7 in addition to 5o. These two ethers $(6,7)$ were adsorbed, when the product mixture was passed through silica with pentane as eluent, and eluted with $\mathrm{CH}_{2} \mathrm{Cl}_{2}$. The 2:1 mixture of 6 and 7 was identified by ${ }^{13} \mathrm{C}-\mathrm{NMR}$ spectroscopy. - 3-(2-Cyclopenten-1-yl)-3-ethoxy-2methyl-1-propene (6): ${ }^{13} \mathrm{C}$ NMR $\left(\mathrm{CDCl}_{3}\right): \delta=15.29\left(\mathrm{q}, \mathrm{OCH}_{2} \mathrm{CH}_{3}\right)$, $16.76\left(\mathrm{q}, \mathrm{CH}_{3}\right), 27.30,31.96$ (2 t, C-3, -4), 48.59 (d, C-3), 63.37 (t, $\left.\mathrm{OCH}_{2} \mathrm{CH}_{3}\right), 88.18(\mathrm{~d}, \mathrm{CH}), 114.11\left(\mathrm{t}, \mathrm{C}=\mathrm{CH}_{2}\right), 131.51,132.08(2 \mathrm{~d}$, $\mathrm{CH}=\mathrm{CH}), 144.45$ (s, $\left.\mathrm{C}=\mathrm{CH}_{2}\right)$. -1 -(2-Cyclopenten-1-yl)-1-ethoxy2-methylpropane (7): ${ }^{13} \mathrm{C} \mathrm{NMR}\left(\mathrm{CDCl}_{3}\right): \delta=15.83\left(\mathrm{q}, \mathrm{OCH}_{2} \mathrm{CH}_{3}\right)$, 18.11, 20.21 [2 q, $\mathrm{CH}\left(\mathrm{CH}_{3}\right)_{2}$ ], 25.15, $32.10(2 \mathrm{t}, \mathrm{C}-4,-5), 31.87$ [d, $\left.\mathrm{CH}\left(\mathrm{CH}_{3}\right)_{2}\right], 48.90$ (d, C-3), 68.27 (t, $\left.\mathrm{OCH}_{2} \mathrm{CH}_{3}\right), 87.84$ (d, $\mathrm{CH}$ ), $131.62,132.87$ ( $2 \mathrm{~d}, \mathrm{C}-1,-2)$.

\section{CAS Registry Numbers}

1a: $109-92-2$ / 1 b: $116-11-0$ / 1c $(E)$ : 4696-26-8 / 1 c (Z): 4696-25-7 1d: $4747-13-1$ / 1 e: $1122-84-5$ / 1 f: $927-61-7$ / 3a: $762-72-1$ / 3b: 18292-38-1 / 3c: $64545-12-6$ / 3d: $58541-14-3$ / 3e: 14579-08-9 / 4a: 22089-55-0 / 4 b: $135312-62-8 / 4 \mathrm{c}\left(R^{*}, R^{*}\right): 135312-63-9 / 4 \mathrm{c}\left(R^{*}, S^{*}\right)$ : 135312-79-7 / 4d: $135312-64-0$ / 4e: $135312-65-1$ / 4f: $135312-66-2$ / 4g: $135312-67-3$ / 4h: $135312-68-4$ / 4i: $135312-69-5$ / 4j: 135312 70-8 / 4k: 135312-71-9 / 4l: 135312-72-0 / 4m: 135312-73-1 / 4n: 135312-74-2 / 4o: 135312-75-3 / 5a: 591-93-5 / 5b: 763-30-4 / 5c (E): 7319-00-8 / 5c (Z): 7318-67-4 / 5d: 35342-69-9 / 5e: 1351113-2 / 5f: 763-88-2 / 5g: 4161-65-3 / 5h: 52713-62-9 / 5i: 13531276-4 / 5j: $927-97-9$ / 5k: 756-02-5 / 5l (E): 135312-77-5 / 51 (Z): 135312-80-0 / 5 m: 135312-78-6 / 5n: 119946-62-2 / 5o: 16839-59-1

\footnotetext{
Dedicated to Professor Karl Heinz Büchel on the occasion of his 60th birthday.

1) See references $2-6$ ) in ref. $^{2 \text { ) }}$
} 
2) B. H. Lipshutz, T. R. Elworthy, J. Org. Chem. 55 (1990) 1695.

3) H. Mayr, W. Striepe, J. Org. Chem. 50 (1985) 2995.

4) For a comprehensive literature report on Lewis acid-catalyzed reactions of $\alpha$-halo ethers with allylsilanes see: I. Fleming, J. Dunoguès, R. Smithers, Org. React. 57 (1989) 57.

5) H. B. Dykstra, J. F. Lewis, C. E. Boord, J. Am. Chem. Soc. 52 (1930) 3396.

6) For related eliminations with $\mathrm{Na}$ in ether solvents see: ${ }^{6 a)} \mathrm{H}$. O. House, R. S. Ro, J. Am. Chem. Soc. 80 (1958) 182. - ${ }^{6 b)}$ R. Paul, O. Riobé, M. Maumy, Org. Synth., Coll. Vol. VI (1988) 675.

7) $M$. Schlosser in Methoden der organischen Chemie (HoubenWeyl-Müller) Bd. 5/1b, 4. Aufl., p. 212, Georg Thieme Verlag Stuttgart 1972.

8) B. H. Shoemaker, C. E. Boord, J. Am. Chem.. Soc. 53 (1931) 1505

9) $\mathrm{H}$. Meerwein in Methoden der organischen Chemie (HoubenWeyl-Müller), Bd. 6/3, 4. Aufl., p. 95, Georg Thieme Verlag Stuttgart 1965.
10) B. Irrgang, H. Mayr, Tetrahedron 47 (1991) 219.

11) G. Hagen, H. Mayr, J. Am. Chem. Soc. 113 (1991) 4954.

${ }^{12)}$ M. S. Wrighton, M. A. Schroeder, J. Am. Chem. Soc. 96 (1974) 6235.

13) T. Hayashi, Y. Katsuro, M. Kumada, Tetrahedron Lett. 21 (1980) 3915.

14) D. C. Rowlands, K. W. Greenlee, J. M. Derfer, C. E. Boord, J. Org. Chem. 17 (1952) 807.

15) Y. Frangin, M. Gaudemar, Bull. Soc. Chim. Fr. 1976, 41.

16) J. Barluenga, M. Yus, J. M. Concellon, P. Bernad, J. Chem. Res. (M) 1980,677 .

17) Y. Tajima, E. Kunioka, J. Chem. Soc., Chem. Commun. 1968, 603.

${ }^{18)}$ N. F. Cywinski, J. Org. Chem. 30 (1965) 361.

19) R. C. Larock, W. H. Gong, J. Org. Chem. 54 (1989) 2047.

[220/91] 